\title{
Rare B Decays at the B-Factories
}

\section{Mikihiko Nakao*}

KEK, High Energy Accelerator Research Organization, Tsukuba, Japan

E-mail: mikihiko.nakao@kek.jp

In the spirit of searching physics beyond the Standard Model, latest results on radiative and electroweak penguin decays and tauonic decays of $B$ mesons from Belle and BaBar are reviewed. The covered results are, in the former, $B \rightarrow X_{s} \gamma$ from Belle $B \rightarrow K^{(*)} \ell^{+} \ell^{-}$from Belle and BaBar, $B \rightarrow X_{s} \ell^{+} \ell^{-}$from Belle, and in the latter, $B^{+} \rightarrow \tau^{+} v$ and $B \rightarrow D^{(*)} \tau^{+} v$ from Belle and BaBar.

12th International Conference on B-Physics at Hadron Machines - BEAUTY 2009 September 07 - 112009

Heidelberg, Germany

${ }^{*}$ Speaker. 


\section{Introduction}

Two B-factories, Belle and BaBar, have accumulated unprecedentedly large samples of $B$ meson decays in the last ten years, and have studied $\mathrm{CP}$ violation and rare $B$ meson decays in great detail. After the confirmation of $\mathrm{CP}$ violation in $B$ meson decays as predicted by the KobayashiMaskawa mechanism, the focus of the B-factories is now set on searches for physics beyond the Standard Model (SM). Besides the further measurements of CP violation, two classes of rare $B$ meson decays are considered to be the most suitable for such studies. The first class is the radiative and electroweak penguin decays, in which a photon or a lepton pair is emitted from a loop diagram of a top quark and a $W$ boson, or possibly from a loop of hypothetical higher mass particles (Figs. 1a-c). The second class includes those involve a $\tau$ lepton in the final state, and are sensitive to a non-SM charged Higgs boson (Figs. 1d-e). In this review, latest results from Belle and BaBar on these decay channels and their implications are discussed.

\section{Radiative and Electroweak Penguin Decays}

In the SM, the dominant contribution to the $b \rightarrow s \gamma$ transition is described by the magnetic penguin operator $O_{7}$ and the Wilson coefficient $C_{7}$. As inclusive $B \rightarrow X_{s} \gamma$ branching fraction is proportional to $\left|C_{7}\right|^{2}$ at the leading order, it is sensitive to physics beyond the SM in terms of possible deviation in $\left|C_{7}\right|$ from the SM value. The $b \rightarrow s \ell^{+} \ell^{-}$transition is similarly described including two more vector and axial-vector electroweak operators $O_{9}$ and $O_{10}$, and corresponding Wilson coefficients $C_{9}$ and $C_{10}$. The transition amplitude is a function of the momentum squared $q^{2}$ carried by the virtual photon or weak boson, which is measured as the square of the invariant mass of the dilepton system. The differential inclusive $B \rightarrow X_{S} \ell^{+} \ell^{-}$branching fraction, which is written down (using $\hat{s}=q^{2} / m_{B}^{2}$ ) as

$$
\begin{aligned}
& \frac{d \Gamma\left(B \rightarrow X_{s} \ell^{+} \ell^{-}\right)}{d \hat{s}}=\left(\frac{\alpha_{\mathrm{em}}}{4 \pi}\right)^{2} \frac{G_{F}^{2} m_{b}^{5}\left|V_{t s}^{*} V_{t b}\right|^{2}}{48 \pi^{3}}(1-\hat{s})^{2} \\
& \times\left[(1+2 \hat{s})\left(\left|C_{9}\right|^{2}+\left|C_{10}\right|^{2}\right)+4\left(1+\frac{2}{\hat{s}}\right)\left|C_{7}\right|^{2}+12 \operatorname{Re}\left(C_{7} C_{9}\right)\right]+\text { corr. }
\end{aligned}
$$
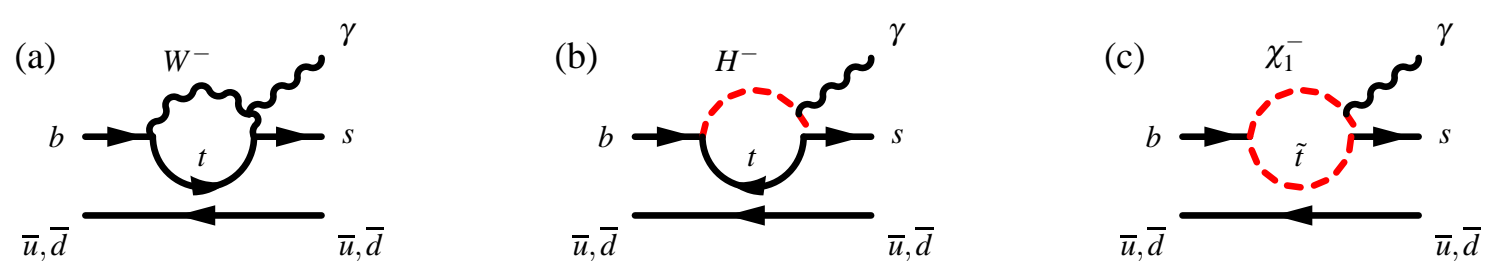

(d)

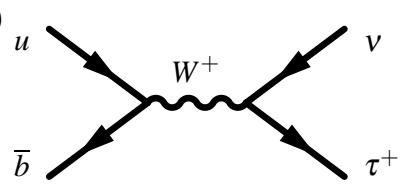

(e)

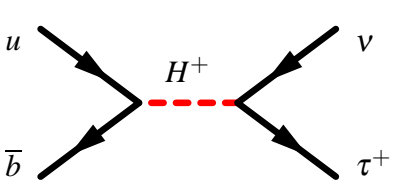

Figure 1: Examples of Feynman diagrams for the $b \rightarrow s \gamma$ transition in the SM (a) and beyond (b,c), and for $B^{+} \rightarrow \tau^{+} v$ in the SM (d) and beyond (e). 
would eventually allow us to disentangle these three Wilson coefficients if sufficient statistics are provided. Meanwhile, the forward backward asymmetry of $B \rightarrow K^{*} \ell^{+} \ell^{-}$,

$$
A_{\mathrm{FB}}\left(B \rightarrow K^{*} \ell^{+} \ell^{-} ; q^{2}\right)=-C_{10} \xi\left(q^{2}\right)\left[\operatorname{Re}\left(C_{9}\right) F_{1}+\frac{1}{q^{2}} C_{7} F_{2}\right],
$$

where $\xi$ and $F_{1,2}$ are known functions, can be used to extract the Wilson coefficients in a different form, from exclusive decay channels that are much easier to measure.

There are more observables that will not be discussed here. In $b \rightarrow s \gamma$, the photon energy spectrum is used to extract the heavy quark parameters that are needed to determine $V_{u b}$, and timedependent CP violation of certain exclusive final states is measured to search for non-SM righthanded current. The $b \rightarrow s \ell^{+} \ell^{-}$process provides even more rich set of observables due to its three-body decay nature and multiple final state particle species.

\section{1 $B \rightarrow X_{s} \gamma$ branching fraction}

The $B \rightarrow X_{s} \gamma$ event can be identified by tagging the high energy photon, which is monochromatic in the $B$ meson rest frame if QCD corrections are absent. In reality the photon energy spectrum has a long tail. The larger coverage of the low energy photons is the key to reduce the theoretical error, while it is a big challenge since the background becomes extremely overwhelming.

The largest (second largest) background is due to $\pi^{0} \rightarrow \gamma \gamma(\eta \rightarrow \gamma \gamma)$ in which one of the photons is undetected. The dominant source is the continuum light quark pair production $e^{+} e^{-} \rightarrow$ $q \bar{q}$, which can be safely subtracted by using data taken below the $\Upsilon(4 S)$ resonance. The contribution from $B$ decays is inferred by positively measuring the high energy $\pi^{0}$ and $\eta$ spectra from $B$ decays, where the $q \bar{q}$ contribution to them is similarly subtracted. One of the possible option to reduce $q \bar{q}$ background is to tag a lepton in the event. Since it reduces the signal at the same time, the net effect is comparable with the untagged case.

Belle has published the latest measurement of $B \rightarrow X_{s} \gamma$ using both tagged and untagged methods on a sample of $657 \times 10^{6} B$ meson pairs [1]. Thanks to the larger statistics and improved techniques, the photon energy threshold was lowered to $1.7 \mathrm{GeV}$, which can be compared with Belle's previous result with $1.8 \mathrm{GeV}$ or BaBar's result with $1.9 \mathrm{GeV}$. The photon energy spectra for the untagged sample, lepton-tagged sample and their average are shown in Fig. 2. The branching fraction was measured to be

$$
\mathscr{B}\left(B \rightarrow X_{s} \gamma\right)=(3.45 \pm 0.15 \pm 0.40) \times 10^{-4}\left(\text { for } E_{\gamma}>1.7 \mathrm{GeV}\right)
$$

According to the prescription by HFAG [2], the result covers about $98.5 \%$ of the branching fraction for $E_{\gamma}>1.6 \mathrm{GeV}$ with which the comparison to theory predictions are usually made. This result is in agreement with the SM prediction, which is $(3.15 \pm 0.23) \times 10^{-4}$ for $E_{\gamma}>1.6 \mathrm{GeV}$, and it alone provides a charged Higgs mass lower bounds of $260 \mathrm{GeV}$ [3]. The world average by HFAG becomes $(3.57 \pm 0.24) \times 10^{-4}$ for $E_{\gamma}>1.6 \mathrm{GeV}$.

\section{$2.2 B \rightarrow K^{*} \ell^{+} \ell^{-}$forward backward asymmetry}

Exclusive decay channel $B \rightarrow K^{*} \ell^{+} \ell^{-}$is reconstructed by combining the momenta of all final state particles. Since Belle and $\mathrm{BaBar}$ are capable to measure and distinguish charged $\pi, K, e$ and 

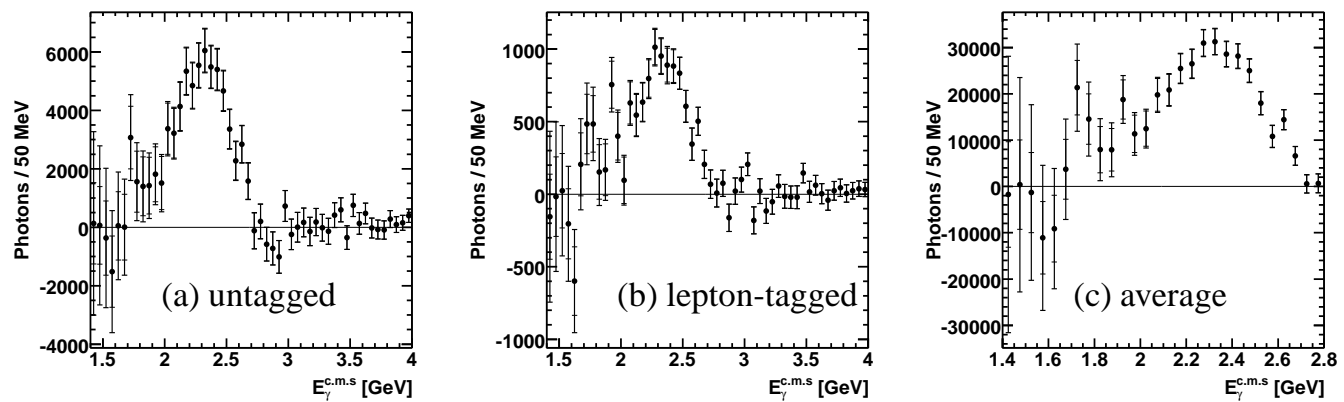

Figure 2: Background subtracted photon energy spectra by Belle for the (a) untagged and (b) lepton-tagged samples, and (c) their efficiency corrected average.
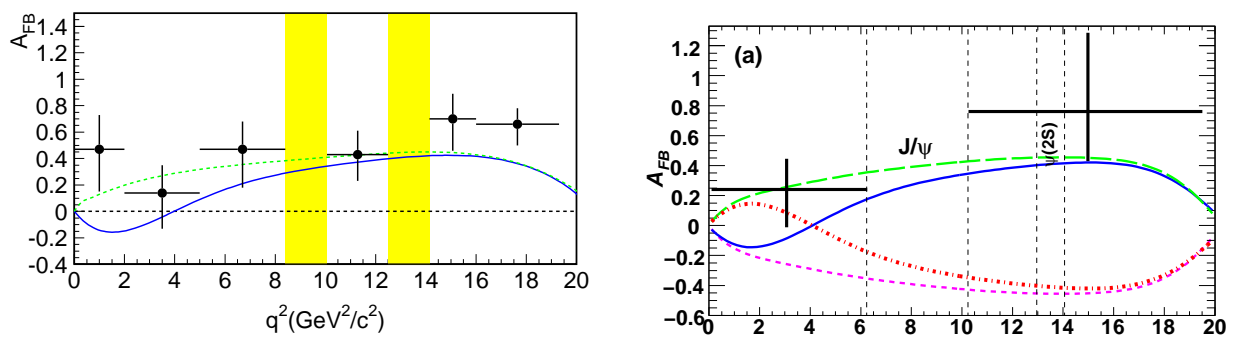

Figure 3: Forward-backward asymmetry of $B \rightarrow K^{*} \ell^{+} \ell^{-}$by Belle (left) and BaBar (right), compared with the SM (solid) and the cases with the flipped sign of $C_{7}$ (dashed in left, long-dashed in right) or the flipped sign of $C_{9}$ (short-dashed and dot-dashed in right).

$\mu$, and neutral $\pi^{0} \rightarrow \gamma \gamma$ and $K_{S}^{0} \rightarrow \pi^{+} \pi^{-}$, all of the following decay channels of $B \rightarrow K^{*} \ell^{+} \ell^{-}$, where $K^{*}$ is $K^{* 0} \rightarrow K^{+} \pi^{-}, K^{*+} \rightarrow K^{+} \pi^{0}$ or $K^{*+} \rightarrow K_{S}^{0} \pi^{+}$and $\ell^{+} \ell^{-}$is $e^{+} e^{-}$or $\mu^{+} \mu^{-}$, can be precisely reconstructed using kinematic variables $M_{\mathrm{bc}} / M_{\mathrm{ES}}$ and $\Delta E$. Although their branching fractions are not precisely interpreted due to theoretical uncertainties on form factors, various ratios and asymmetries such as the forward-backward asymmetry $A_{\mathrm{FB}}$, longitudinal polarization fraction $F_{L}$, isospin asymmetry and lepton flavor asymmetries are good observables. The kaon and lepton decay angles $\theta_{K}$ and $\theta_{\ell}$ in the $K^{*}$ and $\ell^{+} \ell^{-}$rest frame, respectively, distribute according to

$$
\begin{gathered}
\frac{3}{2} F_{L} \cos ^{2} \theta_{K}+\frac{3}{4}\left(1-F_{L}\right)\left(1-\cos ^{2} \theta_{K}\right) \\
\frac{3}{4} F_{L}\left(1-\cos ^{2} \theta_{\ell}\right)+\frac{3}{8}\left(1-F_{L}\right)\left(1+\cos ^{2} \theta_{\ell}\right)+A_{\mathrm{FB}} \cos \theta_{\ell} .
\end{gathered}
$$

The measured distributions are shown in Fig. 3 [4, 5]. In both Belle's and BaBar's results, the measured data points are consistent with the SM expectations, but somehow prefer positive values and are more consistent with the case where the sign of $C_{7}$ is flipped from the SM. The Belle data points are deviated from the SM case at the $2.7 \sigma$ significance. 

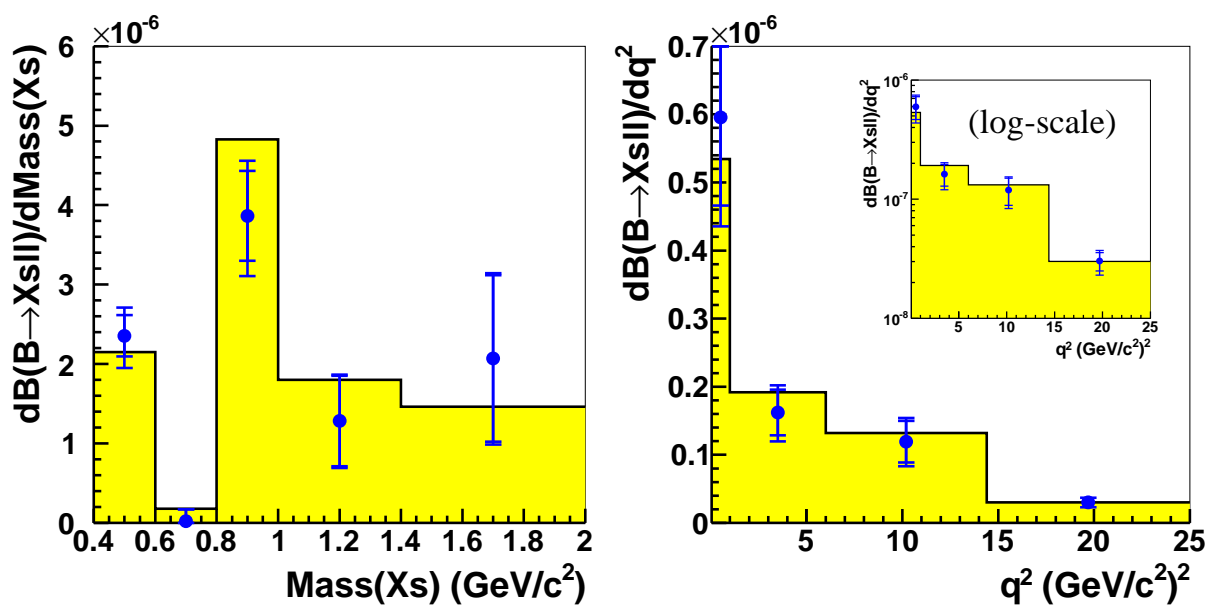

Figure 4: Differential branching fractions of $B \rightarrow X_{s} \ell^{+} \ell^{-}$as functions of $M_{X_{s}}$ (left) and $q^{2}$ (right) by Belle.

\section{$2.3 B \rightarrow X_{s} \ell^{+} \ell^{-}$branching fraction}

Belle has updated the inclusive $B \rightarrow X_{s} \ell^{+} \ell^{-}$branching fraction using 4.5 times more data than previous analysis [6]. The measurement is performed in the sum-of-exclusive decay modes, where $X_{s}$ is a $K^{+}$or $K_{S}^{0}$ combined with up to four pions (of which up to one is $\pi^{0}$ ). A few new sources of backgrounds such as the singly misidentified semileptonic decay chain, $B \rightarrow X_{c}(\ell v) \rightarrow X_{s} \ell v$ are identified. In order to reduce the largest systematic error due to the unknown $X_{s}$ mass spectrum, events with $M_{X_{s}}<2.0 \mathrm{GeV}$ are divided in bins of $M_{X_{s}}$ (Fig. 4). For $M_{X_{s}}>1.0 \mathrm{GeV}$, where already measured $B \rightarrow K^{(*)} \ell^{+} \ell^{-}$contributions are removed, a $3 \sigma$ signal was observed. The result was

$$
\mathscr{B}\left(B \rightarrow X_{s} \ell^{+} \ell^{-}\right)=\left(3.33 \pm 0.80_{-0.24}^{+0.19}\right) \times 10^{-6},
$$

which is for $q^{2}>0.2 \mathrm{GeV}^{2}$ and inter-/extrapolated over the unused $J / \psi$ and $\psi^{\prime}$ mass regions and entire $M_{X_{s}}$ region. The branching fraction, as well as the $q^{2}$ distribution (Fig. 4), is in agreement with the SM expectation, $\mathscr{B}_{\mathrm{SM}}=(4.2 \pm 0.7) \times 10^{-6}$ [7]. The lower central value than the SM implies that the opposite-sign $C_{7}$ is not favored, in contradiction to the results of $A_{\mathrm{FB}}\left(B \rightarrow K^{*} \ell^{+} \ell^{-}\right)$.

\section{Tauonic decays}

Decay modes including a $\tau$ lepton in the final state, $B^{+} \rightarrow \tau^{+} v$ and $B \rightarrow D^{(*)} \tau^{+} v$, are sensitive at the tree level to the non-SM charged Higgs boson. Their measurements are experimentally challenging, since the fully exclusive signal $B$ reconstruction is not possible due to at least two missing neutrinos in the final state. Nevertheless by tagging the other $B$ in the event and selecting the events with a large missing mass, one can deduce the existence of the signal $\tau$ lepton from $B$ decays. This type of measurement has become possible only recently, with a large data sample to compensate the tiny efficiency to tag the other $B$.

Leptonic two-body $B$ decays are helicity suppressed in the SM, to which the charged Higgs boson has a destructive contribution [8],

$$
\mathscr{B}\left(B^{+} \rightarrow \tau^{+} v_{\tau}\right)=\mathscr{B}_{\mathrm{SM}}\left(B^{+} \rightarrow \tau^{+} v_{\tau}\right) \times\left(1-\frac{m_{B}^{2}}{m_{H^{+}}^{2}} \tan ^{2} \beta\right)^{2},
$$


unless $m_{H^{+}} / \tan \beta$ is smaller than $m_{B} / \sqrt{2}$. Eq. 3.1 is universal for all leptons, but the $B^{+} \rightarrow \tau^{+} v$ mode is the most accessible due to the reduced helicity suppression. The SM branching fraction has uncertainties from the decay constant $f_{B}$ and the CKM parameter $\left|V_{u b}\right|$, which could be reduced to that of the bag parameter $B_{B}$ and other better measured CKM parameters by combining with precisely measured $\Delta m_{d}$.

Semileptonic tauonic decays have a more complicated formula. For $B \rightarrow D \tau^{+} v$,

$$
\mathscr{B}(B \rightarrow D \tau v)=G_{F}^{2} \tau_{B}\left|V_{c b}\right|^{2} f\left(F_{V}, F_{S}, \frac{m_{B}^{2}}{m_{H^{+}}^{2}} \tan ^{2} \beta\right),
$$

where $f$ is a function of the same non-SM quantity $\left(m_{B} / m_{H^{+}}\right) \tan \beta$ and form factors $F_{S}$ and $F_{V}$, whose uncertainties can be studied using $B \rightarrow D \ell^{+} v$ decays ( $B \rightarrow D^{*} \tau^{+} v$ has more form factors). Having different sources of theoretical uncertainties, these two measurements are complementary each other, and in addition can be used to test the universality of the $b-u-H^{+}$and $b-c-H^{+}$couplings.

\section{1 $B^{+} \rightarrow \tau^{+} v$ branching fraction}

Thanks to the simple final state, the other $B$ of $B^{+} \rightarrow \tau^{+} v$ can be the hadronic tag $(B \rightarrow$ $D^{(*)} \pi, D^{(*)} \rho$, etc) or $B \rightarrow D^{*} \ell^{+} v$ tag. After tagging the other $B$ and selecting $\tau$ decay products such as $e, \mu, \pi, \pi \pi^{0}$ or $3 \pi$, there should be no remaining activities in the event. This is characterized by the extra energy in the calorimeter, where the signal has a peak at zero while backgrounds are almost uniformly distributed. Both Belle and BaBar have performed both methods, and obtained very similar results $[9,10,11,12]$. The world average is

$$
\mathscr{B}\left(B^{+} \rightarrow \tau^{+} v\right)_{\mathrm{WA}}=(1.73 \pm 0.35) \times 10^{-4},
$$

which may be compared with an SM prediction $\mathscr{B}_{\mathrm{SM}}\left(B^{+} \rightarrow \tau^{+} v\right)=(1.20 \pm 0.25) \times 10^{-4}$ where $f_{B}$ is from HPQCD [13] and $\left|V_{u b}\right|$ is from HFAG [2]. In a less model dependent prediction using CKM parameters, $\mathscr{B}_{\mathrm{CKM}}\left(B^{+} \rightarrow \tau^{+} v\right)=\left(0.786_{-0.083}^{+0.179}\right) \times 10^{-4}$ [14], which is $2.4 \sigma$ lower than the world average.

\section{2 $B \rightarrow D^{(*)} \tau^{+} v$ branching fractions}

The largest background to $B \rightarrow D^{(*)} \tau^{+} v$ is $B \rightarrow D^{(*)} \ell^{+} v$, which could be distinguished mainly by the missing mass squared $\left(\mathrm{MM}^{2}\right)$, and the tagging method of the other $B$ is limited to the hadronic tag. The lepton momentum $\left(p_{\ell}\right)$ also provides some discrimination, and the extra energy $\left(E_{\text {extra }}\right)$ is still useful to suppress other backgrounds. Belle uses $\mathrm{MM}^{2}$ and $E_{\text {extra }}$, while BaBar uses $\mathrm{MM}^{2}$ and $p_{\ell}$ to extract the signal events.

Since there are large feed across components between the $D^{*}$ modes and $D$ modes, and between the $D^{*+}$ mode and $D^{0}$ mode, all four modes $\left(D^{0}, D^{+}, D^{* 0}\right.$ and $\left.D^{*+}\right)$ are simultaneously fitted. The results are normalized to the corresponding $B \rightarrow D^{(*)} \ell^{+} v$ branching fractions to reduce various systematic errors. All modes are measured with more than $3 \sigma$ significance at least either by Belle or BaBar $[15,16]$. The world average for the ratio of the branching fractions for combined $B \rightarrow D \tau^{+} v$ is,

$$
\left[\mathscr{B}\left(B \rightarrow D \tau^{+} v\right) / \mathscr{B}\left(B \rightarrow D \ell^{+} v\right)\right]_{\mathrm{WA}}=(49 \pm 10) \%
$$




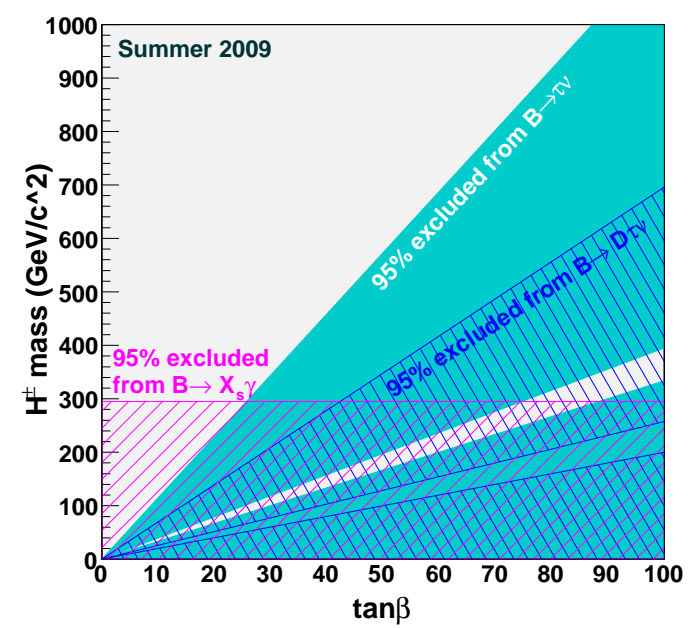

Figure 5: Charged Higgs mass bound as a function of $\tan \beta$ from $B \rightarrow X_{s} \gamma, B^{+} \rightarrow \tau^{+} v$ and $B \rightarrow D \tau^{+} v$.

which can be compared with the SM expectation of $(31 \pm 2 \%)$ [17]. The result is similar in $B \rightarrow$ $D^{*} \tau^{+} v$. This result provides a charged Higgs exclusion region which is complementary to that from $B^{+} \rightarrow \tau^{+} v$.

\section{Summary}

Rare $B$ meson decay modes, $B \rightarrow X_{s} \gamma, B \rightarrow K^{*} \ell^{+} \ell^{-}, B \rightarrow X_{s} \ell^{+} \ell^{-}, B^{+} \rightarrow \tau^{+} v, B \rightarrow D \tau^{+} v$ have been measured and used to provide interesting constraints on physics beyond the SM. For example, 2HDM Charged Higgs bound from $b \rightarrow s \gamma, B^{+} \rightarrow \tau^{+} v$ and $B \rightarrow D \tau^{+} v$ (Fig. 5) is already comparable or better than that expected at LHC [18]. Wilson coefficients $C_{7}, C_{9}$ and $C_{10}$ also constrain various types of new physics.

In the currently available data, there are two possibly interesting signatures. The first is the $\mathscr{B}\left(B^{+} \rightarrow \tau^{+} v\right)$ which may differ from the expectation from the CKM parameters, and the second is the sign of $C_{7}$ from forward-backward asymmetry of $B \rightarrow K^{*} \ell^{+} \ell^{-}$. Final Belle/BaBar data samples are yet to be analyzed, and we anticipate more interesting results at Super B factories.

\section{References}

[1] Belle Collaboration, A. Limosani et al., Phys. Rev. Lett. 103, 241801 (2009).

[2] Heavy Flavor Averaging Group, E. Barberio et al., arXiv:0808.1297 and online update at http://www.slac.stanford.edu/xorg/hfag.

[3] M. Misiak et al., Phys. Rev. Lett. 98, 022002 (2007).

[4] Belle Collaboration, J.-T. Wei et al., Phys. Rev. Lett. 103, 171801 (2009).

[5] BaBar Collaboration, B. Aubert et al., Phys. Rev. D 79, 031102R (2009).

[6] Belle Collaboration, in talk given by T. Iijima at XXIV International Symposium on Lepton Photon Interactions, Jul., 2009. 
[7] A. Ali et al., Phys. Rev. D 66, 034002 (2002).

[8] W.-S. Hou, Phys. Rev. D 48, 2342 (1993).

[9] Belle Collaboration, K. Ikado et al., Phys. Rev. Lett. 97, 251802 (2007).

[10] Belle Collaboration, I. Adachi et al., arXiv:0809.3834v1 [hep-ex].

[11] BaBar Collaboration, B. Aubert et al., Phys. Rev. D 77, 011107 (2008).

[12] BaBar Collaboration, B. Aubert et al., arXiv:0809.4027v1 [hep-ex].

[13] HPQCD Collaboration, E. Gámiz et al., Phys. Rev. D 80, 014503 (2009).

[14] CKMfitter Group, J. Charles et al., Eur. Phys. J. C 41, 1 (2005) and online update at http://ckmfitter.in2p3.fr.

[15] Belle Collaboration, I. Adachi et al., arXiv:0910.4301v1 [hep-ex].

[16] BaBar Collaboration, B. Aubert et al., Phys. Rev. Lett. 100, 021801 (2008).

[17] H.Itoh et al., Prog. Theor. Phys. 114, 179 (2005) with form factors from S. Trine, arXiv:0810.3633 [hep-ph].

[18] ATLAS Collaboration, in talk given by M. Flechl at Workshop on Prospects for Charged Higgs Discovery at Colliders, Sep., 2008. 